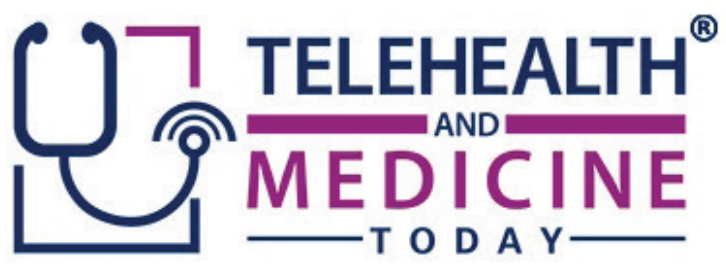

\title{
TMT Editorial: The Future of Virtual Health - Near Term Analysis of the COVID-19 Pandemic
}

\author{
Lyle Berkowitz, MD; ${ }^{1}$ Steve Ommen, MD; ${ }^{2}$ John Halamka, MD ${ }^{3}$
}

Affiliations: ${ }^{1}$ CEO of Back9 Healthcare Consulting, USA; Feinberg School of Medicine at Northwestern University, Chicago, Illinois USA; ${ }^{2}$ Mayo Clinic, Rochester, Minnesota, USA; ${ }^{3}$ President, Mayo Clinic Platform, USA

Corresponding author: Lyle Berkowitz, lyle@drlyle.com

Keywords: COVID-19, Healthcare providers, Pandemic, Telehealth

Section: Editorial

$\mathrm{T}$ elehealth has received a lot of attention these past few months as healthcare providers necessarily shifted a majority of care delivered from onsite to online care almost overnight in mid-March in response to the COVID-19 pandemic. This was initiated by the need to decrease virus exposure and PPE usage, and then further enabled by protelehealth reimbursement and regulatory changes. ${ }^{1}$ What lessons were learned that help us envision what the future might look like over the next 12 months?

First, it is clearly possible for U.S. providers to deliver a significant amount of initial and follow up care via telehealth (real time phone and video consults). The real barriers have been inertia and reimbursement rather than technical or clinical issues. The data is now clear, we can do this! In fact, over $50 \%$ of all billable visits in mid-April of this year were done via telehealth according to
U.S. medical claims data gathered and presented by the consulting company The Chartis. ${ }^{2}$

Of course, the $50 \%$ number can be partially discounted by the fact that total visit numbers were down significantly. In fact, an analysis in a May, 2020 Commonwealth Fund report stated that the telehealth peak in April was closer to $14 \%$ when compared to pre-COVID baseline levels. ${ }^{3}$

However, it is also likely many providers were giving away some amount of care without billing at that time due to uncertainties about reimbursement, technical issues, and other factors. All things considered, we believe that approximately $20-30 \%$ of billable ambulatory care will be done via telehealth through the rest of the year and that percent will then start rising as providers figure out how to do it more efficiently and cost-effectively than office visits. This is consistent with a June, 2020 McKinsey report which proposed that " 35 percent of home 


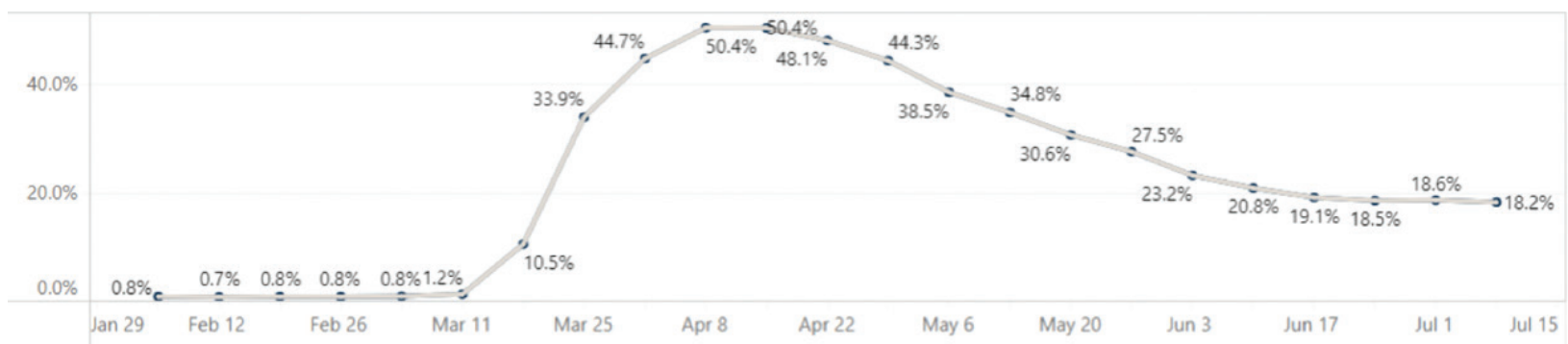

Figure 1-Percent telehealth across all specialties (National) ${ }^{2}$ - Calculated as the volume of telehealth visits as a portion of total visit utilization of all data within the United States (excludes U.S. territories: Puerto Rico, Guam, Virgin Islands).

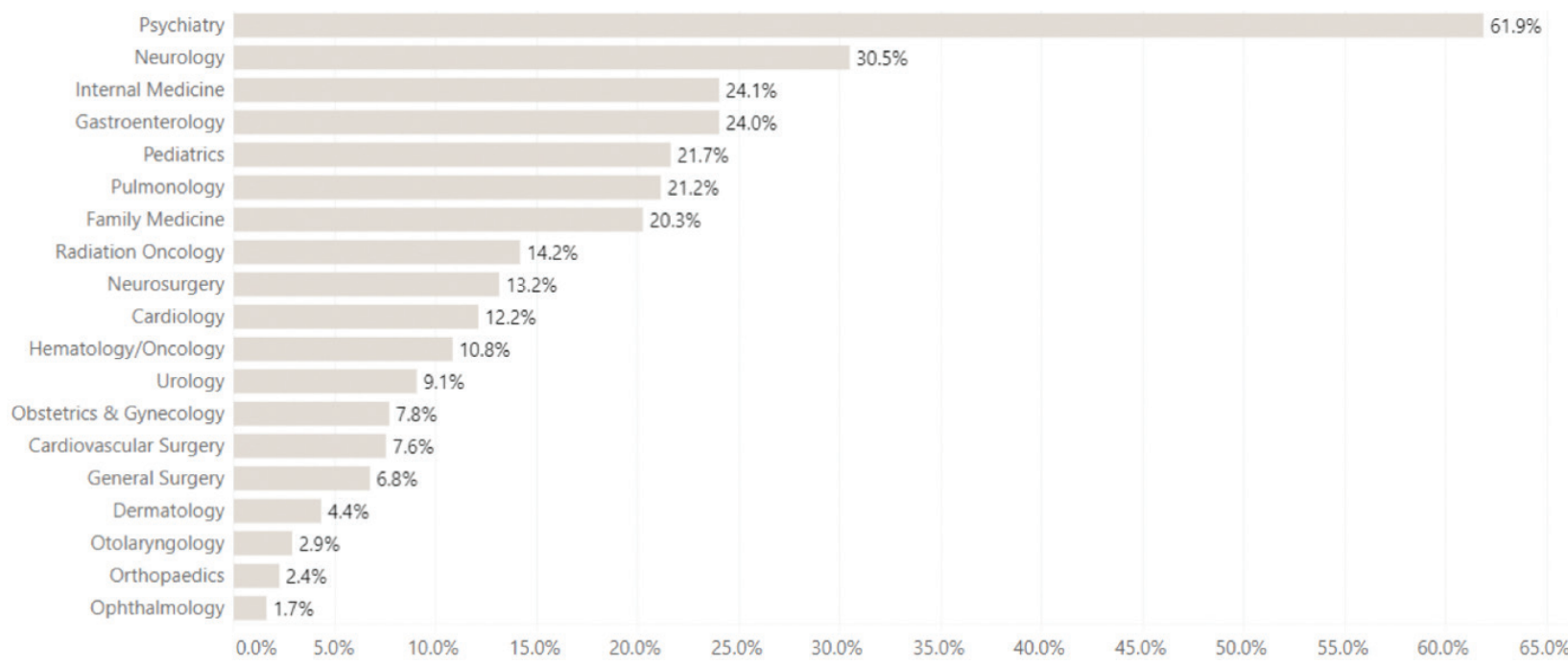

Figure 2-Percentage telehealth by physician specialty (National, week of July 8, 2020)2 - Calculated as the volume of telehealth visits as a portion of total visit utilization of all data within the United States (excludes U.S. territories: Puerto Rico, Guam, Virgin Islands).

health services could move to virtual, as could 24 percent of office visits/outpatient encounters, with another 9 percent moved to 'near virtual', as well as 20 percent of ED visits that can be diverted to virtual". ${ }^{4}$

The potential impediments to this will be whether reimbursement and regulatory reforms are maintained, as well as whether providers can efficiently operationalize their workflows and the new technologies required to be consistently successful.
Second, the amount of telehealth utilization varies widely across specialties. For example, in the chart below, we can see during early July, 2020, that psychiatrists were using telehealth for $61 \%$ of their visits, while other cognitive providers, such as primary care physicians and medical specialists (e.g. neurologists and gastroenterologists, etc.) were doing over $20 \%$ of their care via telehealth. On the other hand, procedure-based specialists were billing under $15 \%$ of their care via telehealth. However, it should be noted that 
many procedural specialists have bundled billing, so they would not charge separately for pre- and post-procedure telehealth calls that are likely now replacing office visits. In the end, this does not change our prediction that at least $20-30 \%$ of all billable care will be done by telehealth in the near term, but that will be a blended rate across specialties. Importantly, this does highlight the need, especially as we expand value-based care reimbursement, to better measure both the activities of providers and the total use of telehealth across various specialties regardless of whether or not it is formally billed.

Third, it is likely that a large amount of the recent telehealth activity was related to urgent care issues, which fortunately prevented a potential landslide of patient visits to immediate care and emergency departments. We believe that patients and doctors have now become increasingly comfortable with doing urgent care visits online and will continue doing them in this manner as per earlier statements. Assuming reimbursement levels remain at parity, we further believe the next big surge of telehealth utilization will involve chronic care and wellness activities as patients try to avoid coming into doctor offices, as home monitoring equipment gets easier and cheaper, and lab testing can be done in highly convenient and efficient locations, including an increasing amount of home testing. Meanwhile, office visits will likely soon get back to normal levels or higher as patients who have been delaying exams or procedures come back into the office, so doctors will need to figure out how to best balance their time. For example, they can alternate visit types throughout the day, or they can set aside half or full days for virtual care. We believe the best use cases among these are to be determined, and will likely vary by specialty, practice type, overall utilization, reimbursement and individual physician comfort level. We may also see organizations merging offices as they recognize some amount of providers can work from home at least a few days a week, and thus they can save on overhead spending via office sharing arrangements.

Finally, we have seen that virtual health will extend beyond traditional telehealth (e.g. synchronous phone and video visits) to also include asynchronous visits and autonomous care.

Asynchronous visits (e.g. text and messaging) are a highly efficient means of communication, although it has not yet received the same reimbursement and regulatory support as synchronous telehealth. We do encourage payors and regulators to more aggressively look at how this can be accomplished. On the other hand, autonomous care (e.g. chatbot, webform input, remote monitoring) is becoming an increasingly important and significant part of our virtual care ecosystem as it has been shown to significantly increase efficiencies and quality in a cost-effective manner. Specifically, we saw that our healthcare system was initially overwhelmed with patients wanting to speak with providers about COVID related questions. The relatively quick implementation of COVID chatbots to answer questions and triage patients to the appropriate level of care, which often meant not talking to a doctor, saved the healthcare system from collapsing on itself. We believe this will open eyes and lay the groundwork for use of similar autonomous care technologies for education, triage, data collection and monitoring for everything from urgent care issues to chronic care problems to wellness activities.

The future of virtual health will thus be a system that uses automation and virtualization to handle routine issues, so that patients with more complex and serious issues can more easily 
get into the office when needed. In other words, the combination of autonomous care and telehealth visits will help ensure we can improve access to high quality, efficient and cost-effective care for more patients across the nation, whether it be autonomous, online or onsite.

Funding Statement: None

\section{Conflict of Interest: None}

Author Contributions: Dr. Berkowitz authored the original drafts. Drs. Ommen and Halamka made revisions in preparation of the final editorial.

\section{REFERENCES}

1. Medicare telemedicine health care provider fact sheet. CMS.gov. 2020. https://www. $\mathrm{cms}$.gov/newsroom/fact-sheets/medicaretelemedicine-health-care-provider-factsheet. Accessed 7/27/70.

2. The Chartis Group and Kythera Labs. Telehealth Adoption Tracker. The Chartis Group. 2020. Available at: https://reports. chartis.com/telehealth_trends_and_ implications/
3. Mehrotra A, Chernew M, Linetsky, Hatch $\mathrm{H}$, Cutler D. The Impact of the COVID19 Pandemic on Outpatient Visits: A Rebound Emerges. The Commonwealth Fund. 2020. Available at: https://www. commonwealthfund.org/publications/2020/ apr/impact-covid-19-outpatient-visits. Accessed 7/27/20.

4. Leventhal R. McKinsey Analysis: Up to \$250B of Healthcare Could Be Virtualized: Researchers note that the shift is not inevitable, however, and the window for providers to act is now. Healthcare Innovation. 2020. Available at: https://www. hcinnovationgroup.com/population-healthmanagement/telehealth/news/21140444/ mckinsey-analysis-up-to-250b-ofhealthcare-could-be-virtualized

Copyright Ownership: This is an open access article distributed in accordance with the Creative Commons Attribution Non Commercial (CC BY-NC 4.0) license, which permits others to distribute, adapt, enhance this work non-commercially, and license their derivative works on different terms, provided the original work is properly cited and the use is noncommercial. See: http://creativecommons. org/licenses/by-nc/4.0. 